\title{
Resistant and disseminated Strongyloides stercoralis infection in a young patient with adult T-cell leukemia/lymphoma
}

\author{
Luís Alberto de Pádua Covas Lage \\ Renata de Oliveira Costa \\ Livia Caroline Barbosa Mariano \\ Erick Menezes Xavier \\ Juliana Pereira
}

Hospital das Clínicas da Faculdade de Medicina, Universidade de São Paulo - USP, São Paulo, SP, Brazil
Conflict-of-interest disclosure:

The authors declare no competing financial interest

\section{Submitted: 6/5/2012}

Accepted: 10/29/2012

\section{Corresponding author:}

Livia Caroline Barbosa Mariano

Departamento de Hematologia - Instituto do Câncer do Estado de São Paulo/Hospital das Clínicas da Faculdade de Medicina da Universidade de São Paulo

Av. Dr. Enéas de Carvalho Aguiar, 155

$1^{\circ}$ andar - SALA 61

05403-900 São Paulo, SP, Brazil

licaroline@gmail.com
Adult T-cell leukemia/lymphoma (ATLL) is a mature T-cell malignancy associated to the human T-cell lymphotropic virus type 1 (HTLV-1). There are between 10 and 20 million HTLV-1 carriers worldwide, particularly in Southwest Japan, the Caribbean, Central Africa, South America and Papua New-Guinea.

In developed countries the median age of patients with ATLL is 60 years, however this is lower in developing countries. In Brazil rare cases have been described in infancy. This earlier incidence of ATLL may be linked to an excess of antigenic stimuli caused by chronic and multiple parasitic infections commonly seen in poor countries ${ }^{(1)}$.

This article reports on an ATLL case in a young woman with massive and refractory Strongyloides stercoralis infection.

A 17-year-old woman came to the Hospital das Clínicas da Faculdade de Medicina da Universidade de São Paulo with abdominal pain and diarrhea that caused a $13 \mathrm{~kg}$ weight loss. Strongyloidiasis, giardiasis and esophageal candidiasis were identified. Despite of several different therapies including specific treatment, the intestinal strongyloidiasis had persisted for one year when the patient returned to the hospital with sepsis and aseptic meningitis and a HTLV1 infection was detected. A computerized tomography showed enlargement of mesenteric and lung hilum lymph nodes and a lymph node biopsy revealed reactive lymphoid hyperplasia. Three months later wart-like lesions were noticed on the hands and right knee and blood counts showed hemoglobin was $14 \mathrm{~g} / \mathrm{L}$, platelet count $156 \times 10^{9} / \mathrm{L}$, the white blood cell count was $5 \times 10^{9} / \mathrm{L}$, lymphocytes were $1.5 \times 10^{9} / \mathrm{L}$ and flower cells were identified in the blood smear. The immunophenotype by flow cytometry showed abnormal CD $3^{\text {dim }}, \mathrm{CD} 4^{\text {bright }}, \mathrm{CD} 25^{\mathrm{dim}}$ and CD7- $\mathrm{T}$-cell lymphocytes (Figure 1). A monoclonal pattern of HTLV-1 insertion into the neoplastic cells was identified by inverse long polymerase chain reaction (ILPCR) (Figure 2). A smoldering ATLL was diagnosed and treatment with 3 million units of interferon alpha twice per week and zidovudine $900 \mathrm{mg} /$ day was started. The patient remained with itching and intermittent diarrhea, despite of the treatment for strongyloidiasis. After one year the disease progressed with neck, axillary and inguinal lymphadenopathy and splenomegaly. Finally, the patient was admitted to the emergency department in a bad health state with cough, fever, tracheobronchitis and hypercalcemia. Regardless of treatment the patient died after four days due to disseminated Strongyloides stercoralis infection.
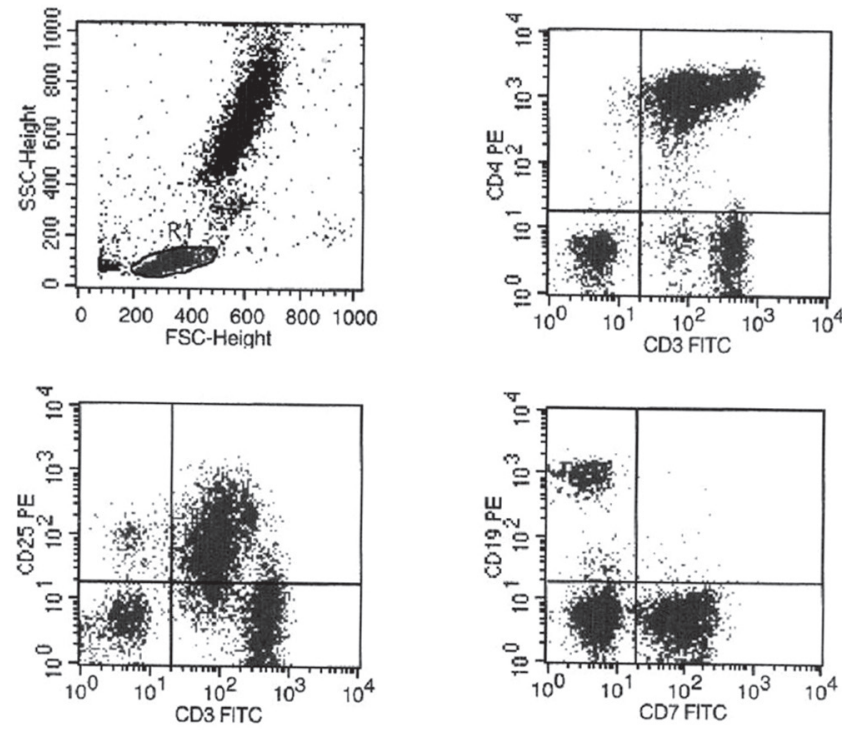

Figure 1 - Flow cytometry analysis 


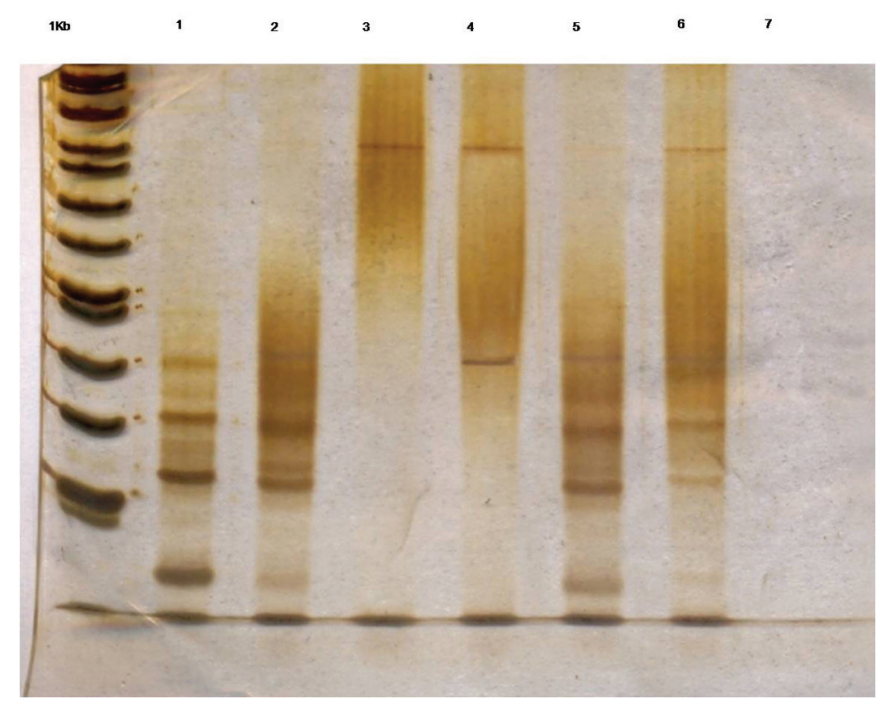

Figure 2 - Polymerase chain reaction diagnostic test

Inverse long polymerase chain reaction in $10 \%$ polyacrylamide gel stained with silver showing in the $1 \mathrm{~Kb}$ line: 1 kilobase ladder; Line 1: human T-cell lymphotropic virus (HTLV) carrier control 1; Line 2: HTLV carrier control 2; Line 3: HTLV negative control; Line 4: adult T-cell leukemia/lymphoma patient; Line 5: HTLV carrier control 3; Line 6: HTLV carrier control 4; Line 7: Amplification control

\section{Discussion}

HTLV-1 belongs to the delta retrovirus group and its pro-viral genome shows gag, pol and env genes and a long terminal repeat region $(L T R)$ at both ends. A codifying region of the viral genome named $p X$ is found between the env gene and 3'-LTR; this region is responsible for the synthesis of the viral regulatory proteins p40 (Tax), p27 (Rex), p12, p13, p21, p30 and b-ZIP factor $(H B Z)$ which are implicated in viral infectivity and proliferation of infected cells. This virus is disseminated by breast feeding, syringe sharing, blood transfusions and sexual intercourse. There is a long latency until ATLL develops and only $3 \%$ to $5 \%$ of HTLV-1 carriers will become sick ${ }^{(2)}$.

After cells are infected by HTLV-1, they form virological synapses with target cells of the host resulting in transference of viral RNA to the target cell. A pro-viral DNA is produced from the viral RNA mediated by the viral reverse transcriptase enzyme. The viral DNA is randomly inserted into the host's DNA. But in ATLL the malignant cells present a monoclonal pattern of HTLV- $1^{(2)}$.

Tax and HBZ are essential in the pathogenesis of ATLL as they induce viral gene expression by the amplification of the LTR and the activation of $N F-K B, S R F, C R E B$ and $A P-1$ transcription signal genes thus preventing expression of the p53 and pl6INK4A anti-oncogenes. These tumor suppressor genes induce T-cell toxicity and reduce the survival of HTLV-1 infected cells. Subsequently Tax is suppressed by epigenetic factors and leukemic cells may escape from immune surveillance and are thus able to proliferate indefinitely. $H B Z$ activates the transcription factor $E 2 F^{(3)}$ and polymorphisms in the HLA A16, $B 4002, B 4006$ and $B 4801$ genes increase the risk of ATLL progression. These polymorphisms are related with a reduced
T-cell function ${ }^{(3)}$. Diagnosis depends of clinical and laboratorial criteria. The laboratorial evaluation includes blood counts, smear analysis and lymphocyte immunophenotype. Eosinophilia is common and related to a worse prognosis. Lymphocytosis with medium-sized flower cells, dense chromatin and nucleus with polylobulated or convolute features with a high degree of basophilia on cytoplasm is the hallmark of acute and chronic ATLL. The common phenotype is CD $3^{\mathrm{dim}}, \mathrm{CD} 4^{\text {bright }}, \mathrm{CD} 25^{\mathrm{dim}}$ and $\mathrm{CD}^{-}$, but a double positive $\mathrm{CD}^{+} / \mathrm{CD}^{+}$ATLL may occur and rare cases may be $\mathrm{CD} 4 / \mathrm{CD}^{+}$.

HTLV-1 must be confirmed by Western-blot with monoclonal viral DNA insertion in lymphocytes being the gold standard for diagnosis ${ }^{(1,2)}$. Bone marrow aspirate and biopsy is not necessary and lymph node biopsy is not enough to make the diagnosis.

The most aggressive and commonest form of the disease is acute ATLL (65\%) characterized by high lymphocyte counts and lactate dehydrogenase (LDH) levels, hepatosplenomegaly, skin lesions and hypocalcemia. The lymphoma form has enlargement of lymph nodes, visceromegaly, normal or high levels of LDH and less than $1 \%$ of neoplastic cells in the blood. Smoldering and chronic ATLL are more indolent; the first may present skin lesions, pulmonary infiltration and a normal lymphocyte count with less than $1 \%$ to $5 \%$ of leukemic cells and normal LDH and calcium. Chronic ATLL has less than 4 x $10^{9} / \mathrm{L}$ lymphocytes and $3 \%$ of circulating flower cells ${ }^{(4)}$.

Skin involvement occurs in $50 \%$ of cases with no specific findings but macula, plaque, nodule, and purpura or erythrodermic eruptions may occur. Macrophage activating syndrome with hemophagocytosis may also be found predicting transformation to more aggressive forms.

Cellular immunity is compromised in ATLL and opportunistic infections with a fatal course such as Pneumocystis jiroveci, cytomegalovirus, Strongyloides stercoralis, Mycobacterium avium and fungi can arise. About $30 \%$ of HTLV-1 carriers with strongyloidiasis show a monoclonal provirus integration suggesting that this parasite may interfere in the pathogenesis of ATLL ${ }^{(5)}$.

ATLL remains incurable and only transitory response can be acquired. The most frequent mechanism of chemotherapy resistance in ATLL is linked to multiple drug resistance (MDR) proteins, mutations of the $p 53$ gene and oncogene abnormalities ${ }^{(4)}$. The aggressive subtypes must be treated but a watch and wait conduct can be followed for indolent forms.

If polychemotherapy is indicated, supportive care with prophylaxis for viral, fungal and bacterial infections should be used as immunodeficiency will be exacerbated by neutropenia secondary to treatment.

Intestinal infection by Strongyloides stercoralis is common before chemotherapy, even in HTLV-1 carriers. Patients with ATLL must be investigated for intestinal parasitosis and eradication of parasitosis with ivermectin or thiabendazole should be performed before chemotherapy to avoid dissemination of the strongyloidiasis.

The most common regimens for aggressive ATLL are anthracycline-based. A study by the Japan Oncology Group observed overall survival (OS) of $66 \%$ [25\% complete remission (CR) and 41\% partial remission (PR)] with CHOP-14. 
The 3-year OS was $12.7 \%$ with 10.9 months of progression free survival (PFS). A sequential aggressive chemotherapy using vincristine, cyclophosphamide, doxorubicin and prednisone; AMP: doxorubicin, ranimustine and prednisone alternated with vindesine, etoposideo, carboplatin and prednisone was better than CHOP-14 for acute lymphoma and unfavorable chronic ATLL (OS: 72\%, CR: $40 \%$ and PR: 32\%; 3-year OS $23.6 \%$ and PFS of 12.7 months). This better OS was related to ranimustine and carboplatin because they are not linked to MDR resistance.

HTLV-1 carriers might not need to be treated and chronic and smoldering ATLL patients do not always need treatment. Smoldering and chronic ATLL can be treated with 6 to 9 million units/day of interferon alpha plus zidovudine $800-1000 \mathrm{mg}$ /day with a 5-year OS of $100 \%{ }^{(6)}$. The better response is seen for wild type $p 53$ and low interferon regulating factor type 4 (IRF-4) patients.

In conclusion, although a rare condition, HTLV-1 infection and ATLL disease should be included in the differential diagnosis of refractory Strongyloides stercoralis infection. In developing countries, a young age might not exclude the diagnosis of ATLL.

\section{References}

1. Pombo de Oliveira MS, Matutes E, Schulz T, Carvalho SM, Noronha H, Reaves JD, et al. T-cell malignancies in Brazil. Clinic-pathological and molecular studies of HTLV-I-positive and-negative cases. Int J Cancer. 1995;60(6):823-7.

2. Yasunaga JI, Matsuoka M. Human T-Cell leukemia virus type I induces adult T-cell leukemia: from clinical aspects to molecular mechanisms. Cancer Control. 2007;14(2):133-40.

3. Shimoyama M. Diagnostic criteria and classification of clinical subtypes of adult T-cell leukemia-lymphoma: A report from the Lymphoma Study Group (1984-87). Br J Haematol. 1991;79(3):428-37.

4. Ratner L. Human $\mathrm{T}$ cell lymphotropic virus-associated leukemialymphoma. Curr Opin Oncol. 2005;17(5):469-73.

5. Matutes E. Adult T-cell leukaemia-lymphoma. J Clin Pathol. 2007;60(12):1373-7.

6. Bazarbachi A, Hermine O, Panelatti G, Ramos JC, Tortevoye P, Otrock Z, Taylor G, Gessain A, Harrington W, Plumelle Y. A worldwide metaanalysis on the use of zidovudine and interferon-alpha for the treatment of adult T-cell leukemia-lymphoma: $13^{\text {th }}$ International Conference on Human Retrovirology; 2008 Jun 12-15. Hakone, Japan. Haematologica. 2008;93(s1):311 abs. 0777 